\title{
Atypical Peritracheobronchial Vasculitis and an Effective Treatment
}

\author{
Naoki Saita, Keiko Asahara, Hirotsugu Kohrogi, Tetsunori SaKata, Moritaka Suga and Masayuki Ando
}

\begin{abstract}
We report an interesting case of vasculitis in which the inflammatory lesion was limited to the peritracheobronchus. This case showed positive antineutrophil cytoplasmic antibodies, diffuse peritracheobronchial swelling, and vasculitis in its histology. Steroid therapy was effective for both roentgenological and serological findings. Although the biopsy specimen shows only inflammation and does not satisfy the WHO criteria of Wegener's granulomatosis (WG), a possible diagnosis of WG should not be disregarded. (Internal Medicine 33: 554-556, 1994)
\end{abstract}

Key words: tracheal swelling, vasculitis, steroid therapy, c-ANCA

\section{Introduction}

We report an atypical peritracheobronchial vasculitis obtained by open chest biopsy. Stein et al reported diffuse tracheal stenosis of Wegener's granulomatosis (WG), and discussed the difficult differential diagnosis such as sarcoidosis, amyloidosis and relapsing polychondritis (1). The present case does not satisfy the WHO criteria for the localized form of WG (2). Several reports discuss difficult and delayed diagnosis of WG $(3,4)$. In one case, the final diagnosis was made at the time of autopsy, thus it is critical not to overlook a clinical diagnosis of WG if the biopsy specimen shows only inflammation (3). In the present case, therefore, considering WG, a close follow-up is necessary. It will also be important to observe tracheal swelling. CT scan was very useful in the detection of the main lesions where steroid therapy was very effective.

\section{Case Report}

A 56-year-old male was admitted with fever $\left(38^{\circ} \mathrm{C}\right)$, tenderness of the parasternal area, and weight loss. There were no abnormal findings in nasopharyngeal lesion, palpable masses, hepatosplenomegaly, or lymph node enlargement. The ESR was $131 \mathrm{~mm} / \mathrm{h}$, CRP $10.4 \mathrm{mg} / \mathrm{dl}$, and no hematuria. Antineutrophil cytoplasmic antibodies (cANCA) were positive $(\times 20)$ (normal, $<\times 10)$. A chest X-ray showed diffuse tracheal thickening but no abnormal shadow in both lung fields. A chest $\mathrm{CT}$ also showed diffuse tracheobronchial thickening (Fig. 1A). Bronchoscopical findings showed slight redness and narrowing of the trachea. Histological examination of a transbronchial biopsy of the carina showed only a slight submucosal edema. As these findings were not sufficient to explain the diffuse tracheal thickening, we performed an open chest biopsy of the peritrachea for histological diagnosis. Peritrachea was smooth and edematous. Histological findings of peritrachea showed vasculitis with plasma cells, neutrophils, macrophages and lymphocytes without showing necrosis (Fig. 2).

As shown in Fig. 3, two days after steroid therapy with prednisolone $(60 \mathrm{mg} / \mathrm{day})$, his complaints of high fever and tenderness of the parasternal area were diminished. ESR and CRP were also improved within 10 days. Furthermore, the thickness of the tracheobronchus was decreased on chest X-ray and CT scan at one month after the therapy (Fig. 1B, Fig. 3).

\section{Discussion}

In the present patient, an atypical vasculitis of inflammatory lesion was found only in the peritracheobronchus. The endobronchial lesion showed marked improvement with prednisolone therapy. As shown in Fig. 3, the patient's complaints of high fever and tenderness of the parasternal area were diminished only two days after prednisolone therapy. The reason for this rapid improvement might be explained by the fact that the main inflammatory lesion was only around the small vascular and the small artery was intact (Fig. 2). So far as we know, this is the first case report of a limited vasculitis with diffuse peritracheobronchial inflammation.

One of the interesting findings of the present case was the

From the First Department of Internal Medicine, Kumamoto University School of Medicine, Kumamoto

Received for publication October 14, 1993; Accepted for publication June 6, 1994

Reprint requests should be addressed to Dr. Naoki Saita, the First Department of Internal Medicine, Kumamoto University School of Medicine, 1-1-1 Honjo, Kumamoto 860 

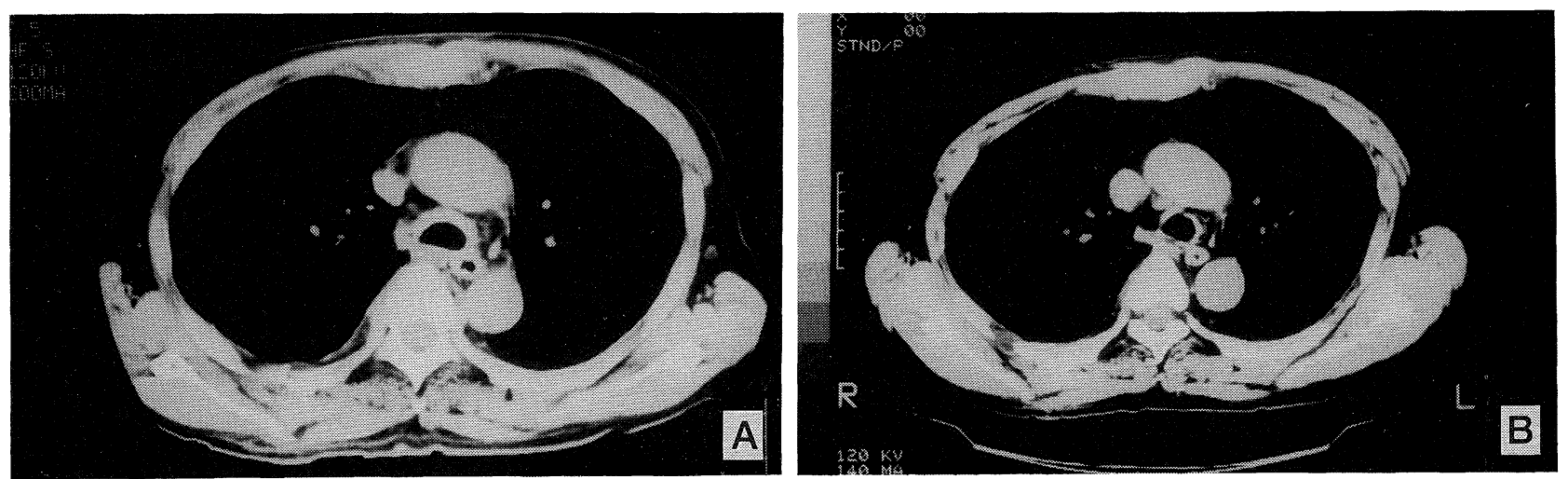

Fig. 1. Chest CT on admission (A), after prednisolone therapy (B).

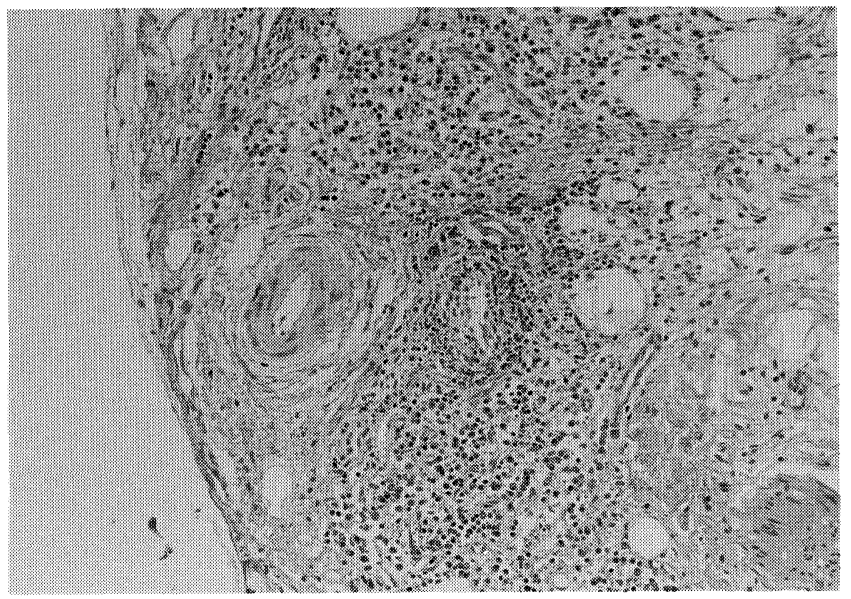

Fig. 2. Biopsy specimen of peritrachea (HE stain, $\times 100)$. positive cANCA test, although the titer was low but significant. This test has been recently recognized as a marker for WG (5) and microscopic polyarteritis vasculitis (6). As the cANCA test turned to negative after prednisolone therapy, cANCA probably participated in the pathogenesis of this disease. Furthermore, this is one of the useful tests for markers of vasculitis activity and treatment effectiveness.

An important diagnostic point of the present case was diffuse tracheal stenosis due to the swelling, which was discussed by Stein et al (1). It is necessary to differentiate from WG. Other granulomatous diseases (e.g. sarcoidosis), amyloidosis, or relapsing polychondritis were thought to be unlikely, since the patient had no other symptoms and signs including histopathological findings.

Typical bronchoscopical findings of WG are redness, swelling, bleeding, engorgement of small vessels, irregularity, ulceration, thickening, and elevated lesion (7). In contrast,
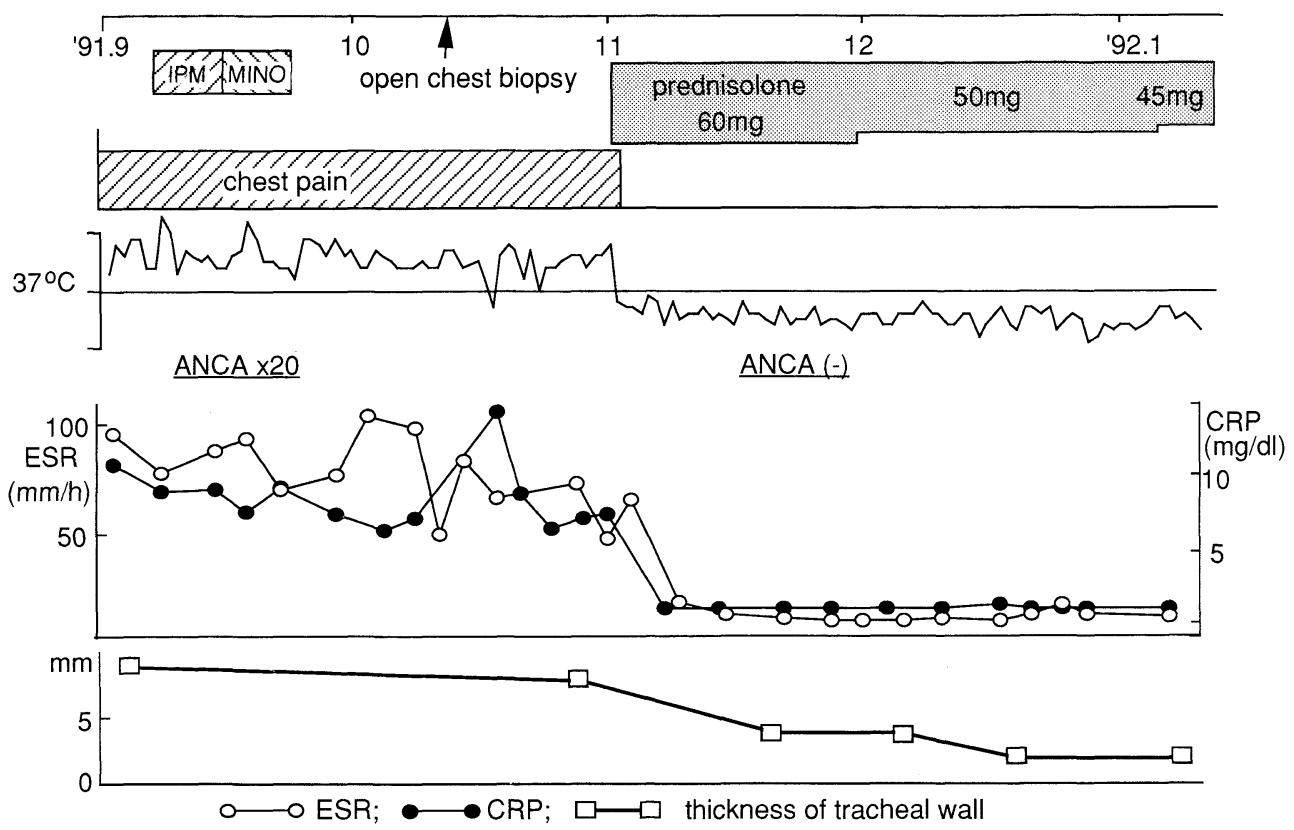

Fig. 3. Clinical course. 
transbronchial biopsies of atypical WG cases have often revealed only nonspecific inflammatory tissue. In all of these cases, nasal or pulmonary biopsy enabled histological diagnosis (8). In the present case, the inflammatory lesion was only in the swelling of the tracheobronchus. Bronchoscopical findings showed slight redness and slight narrowing of trachea. Histological findings of a transbronchial biopsy of the carina showed only submucosal edema.

Although the present case differs from the WHO criteria of WG regarding biopsy findings, Scully et al showed that only irregular focal deposition of fibrin in the lamina propria with intact epithelium and without evidence of vasculitis or granuloma is compatible with and characteristic of early WG (9). Additionally, Shah et al reported an autopsy case of classical vasculitis and granuloma only in the liver and spleen (3). They stressed that, unable to make a final diagnosis until autopsy, it is critical not to negate a possiblity of the clinical diagnosis of WG if the biopsy specimen of a patient with the clinical impression of WG shows only inflammation. In the present case, therefore, considering WG, a close follow-up is necessary with particular consideration to the appearance of additional symptoms or signs such as nasopharyngeal or renal lesion. Observation of tracheal swelling on CT scan was very useful to detect the main lesions, where steroid therapy was very effective.

\section{References}

1) Stein MG, Gamsu G, Webb WR, Stulbarg MS. Computed tomography of diffuse tracheal stenosis in Wegener's granulomatosis. J Comput Assist Tomogr 10: 868, 1986.

2) Shanmugaratnam $\mathrm{K}$, Sobin LH. Histological typing of upper respiratory tract. International Histological Classification of Tumors. No. 19, p51. WHO, Geneva, 1978.

3) Shah IA, Holstege A, Riede UN. Bioptic diagnosis of Wegener's granulomatosis in the absence of vasculitis and granulomas. Pathol Res Pract 178: 407, 1984.

4) Bakheit AMO, Behan. Localised Wegener's granulomatosis. A difficult and delayed diagnosis. Int J Med Science 159: 55, 1990.

5) Van der Woude FJ, Lobatto S, Permin H, et al. Autoantibodies against neutrophils and monocytes: tool for diagnosis and marker of disease activity in Wegener's granulomatosis. Lancet i: 425, 1985.

6) Falk RJ, Jennette JC. Anti-neutrophil cytoplasmic autoantibodies with specificity for myeloperoxidase in patients with systemic vasculitis and idiopathic necrotizing and crescentic glomerulonephritis. N Engl J Med 318: $1651,1988$.

7) Cordier JF, Valeyre D, Guillevin L, Loire R, Brechot JM. Pulmonary Wegener's granulomatosis: A clinical and imaging study of 77 cases. Chest 97: 906, 1990.

8) Aberle DR, Gamsu G, Lynch D. Thoracic manifestations of Wegener's granulomatosis: Diagnosis and course. Radiology 174: 703, 1990.

9) Scully RE, Galdabini JJ, Mcneely BU. Wegener's granulomatosis. N Engl J Med 304: 958, 1990. 